\title{
Profiling in sport using momentum and perturbations
}

\author{
MIKE HUGHES ${ }^{1} \triangle$, PEGGY BÜRGER ${ }^{2}$, MICHAEL T. HUGHES 3 , STAFFORD MURRAY4 ${ }^{4}$ NIC \\ JAMES 1 \\ ${ }^{1}$ London Sport Institute, Middlesex University, London, UK \\ 2 Otto von Guericke Universitat, Magdeburg, Germany \\ ${ }^{3}$ PGIR, Bath, UK \\ ${ }^{4}$ English Institute of Sport, England
}

\section{ABSTRACT}

Hughes $M$, Bürger $P$, Hughes MT, Murray $S$, James $N$. Profiling in sport using momentum and perturbations. J. Hum. Sport Exerc. Vol.8, No. Proc2, pp. S242-S260, 2013. Examining a team's performance from a physical point of view their momentum might indicate unexpected turning points in defeat or success. Physicists describe this value as to require some effort to be started, but also that it is relatively easy to keep it going once a sufficient level is reached (Reed and Hughes, 2006). Unlike football, rugby, handball and many more sports, a regular volleyball match is not limited by time but by points that need to be gathered. Every minute more than one point is won by either one team or the other. That means a series of successive points enlarges the gap between the teams making it more and more difficult to catch up with the leading one. This concept of gathering momentum, or the reverse in a performance, can give the coaches, athletes and sports scientists further insights into winning and losing performances. Momentum investigations also contain dependencies between performances or questions if future performances are reliant upon past streaks. Squash and volleyball share the characteristic of being played up to a certain amount of points. Squash was examined according to the momentum of players by Hughes et al. (2006). The initial aim was to expand normative profiles of elite squash players using momentum graphs of winners and errors to explore 'turning points' in a performance. Dynamic systems theory has enabled the definition of perturbations in sports exhibiting rhythms (Hughes et al., 2000; McGarry et al., 2002; Murray et al., 2008), and how players and teams cause these disruptions of rhythm can inform on the way they play, these techniques also contribute to profiling methods. Together with the analysis of one's own performance it is essential to have an understanding of your oppositions' tactical strengths and weaknesses. By modelling the oppositions' performance it is possible to predict certain outcomes and patterns, and therefore intervene or change tactics before the critical incident occurs. The modelling of competitive sport is an informative analytic technique as it directs the attention of the modeller to the critical aspects of data that delineate successful performance (McGarry \& Franks, 1996). Using tactical performance profiles to pull out and visualise these critical aspects of performance, players can build justified and sophisticated tactical plans. The area is discussed and reviewed, critically appraising the research completed in this element of Performance Analysis. Key words: PROFILING, PERFORMANCE, SPORT, MOMENTUM, PERTURBATIONS.

\footnotetext{
Corresponding author. London Sport Institute, Middlesex University, Houghton Street, London WC2A 2AE, UK

E-mail: mikehughes@data2win.org

7th INSHS International Christmas Sport Scientific Conference, 9-12 December 2012. International Network of Sport and Health Science. Szombathely, Hungary.

JOURNAL OF HUMAN SPORT \& EXERCISE ISSN 1988-5202

(c) Faculty of Education. University of Alicante

doi:10.4100/jhse.2012.8.Proc2.27
} 


\section{INTRODUCTION}

Squash was first examined according to the momentum of players by Hughes et al. (2006), their initial aim was to expand normative profiles of elite squash players using momentum graphs of winners and errors to explore 'turning points' in a performance. Together with the analysis of one's own performance it is essential to have an understanding of your oppositions' tactical strengths and weaknesses. By modelling the oppositions' performance it is possible to predict certain outcomes and patterns, and therefore intervene or change tactics before the critical incident occurs. The modelling of competitive sport is an informative analytic technique as it directs the attention of the modeller to the critical aspects of data that delineate successful performance (McGarry \& Franks, 1996). Using tactical performance profiles to pull out and visualise these critical aspects of performance, players can build justified and sophisticated tactical plans.

Acknowledgment of the importance of notational analysis to improving performance was best demonstrated by the appointment of full-time analysts with the England and Scottish squash squads. Indeed, Murray \& Hughes, (2001) showed how modern technology and their application can provide tactical profiles of players and their opponents. These profiles are not dissimilar to those pioneered by Hughes (1985), and extended by Hughes \& Robertson (1998), but are more soundly based in terms of reliability and have the requisite amounts of data to create stable profiles.

The research of Hughes et al. (2006) aimed to extend these ideas and explore a new application of these analysis techniques to develop 'momentum profiles' through notational analysis. They defined positive momentum as a player hitting successive winners, negative momentum, as a player hitting errors, and if the player is passive, not hitting winners or making errors, then the player's momentum will stay the same. This work was a first step in trying to apply these ideas of momentum within a game and correlate them with overall performance in the elite playing world.

The momentum curves all show interesting aspects of players' performances - in Figure 1, for example Sarah Fitzgerald was playing 'steadily' for the first 2 games but, round about rally 10 of game 3 , something happened and she suddenly started playing much more positively, and she sustained this to win the match. What happened at this point in the game? Examining more graphs like this one, it seemed that players had positive and negative swings in momentum. Do these cycles occur regularly and do they have repetitive properties?

\section{MATERIAL AND METHODS}

Momentum graphs - the beginning in squash

The initial data collection used a real-time analysis system which is based on a program designed by Hughes \& Robertson (1995) and later computerised to speed up the process (Murray \& Hughes, 2001). This system provides an analysis of winners and errors, gathering data such as, the number of shots in a rally, the position from which the rally ending shot was hit, who hit it, and the type of rally ending shot. By writing another analysis program we calculated a running score (momentum) for a player during a game. We gave a winning shot by a player a ' +1 ' score, an error a ' -1 ' score, and if the opponent hit the rally end shot, or it was a let, the running score stayed the same (Figure 1). This could just as easily be done with scoring, or positive and negative phases of play, in any team game (Reed \& Hughes, 2006; Locke, 2005). With the data processed by the software it was possible to present the 2 sets of data for both players at the same time, see figures 1 and 2 . It is possible to examine these curves and see where the 'turning points' in 
the match occurred. For the coach and/or the team psychologist to discuss with the player what happened at these turning points would enhance insight into each respective performance.

Figure 3 shows another way that we could present these data; we termed this form of presentation a 'cumulative' momentum graph. In this graph the momentum of each player 'interacts' - so that a winner by a player will move the graph upwards, whereas an error by the player moves it downwards. The opposite applies to the other player - a winner moving the graph downward and an error moving it upwards. In this way one curve represents the interactive momentum of the match, with each player having their positive areas on each side of the abscissa and the turning points can be seen clearly.

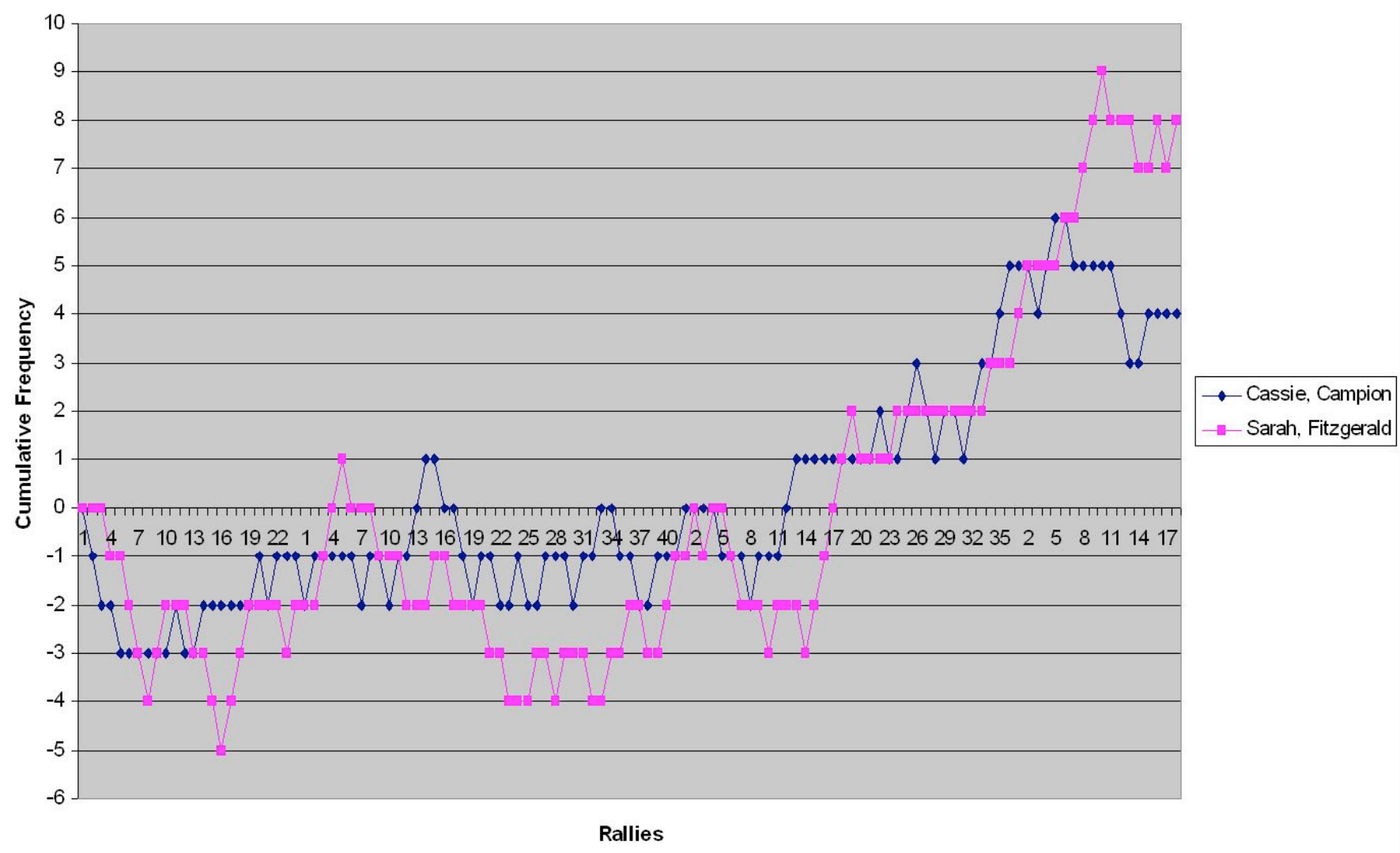

Figure 1. Example of 'momentum analysis' graph 


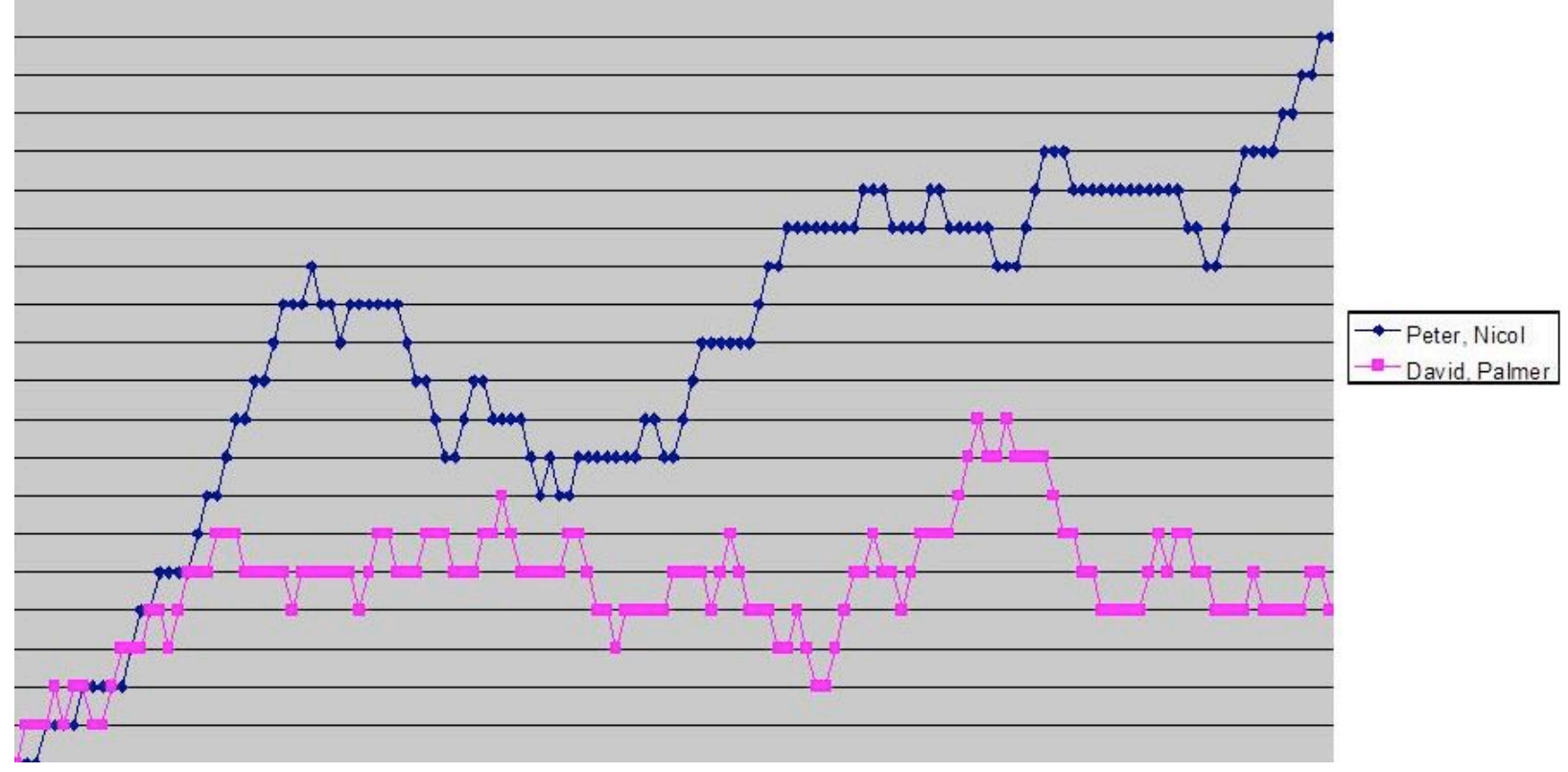

Figure 2. Example of 'momentum analysis' graph

Momentum Theory - Player 1 v Player 2

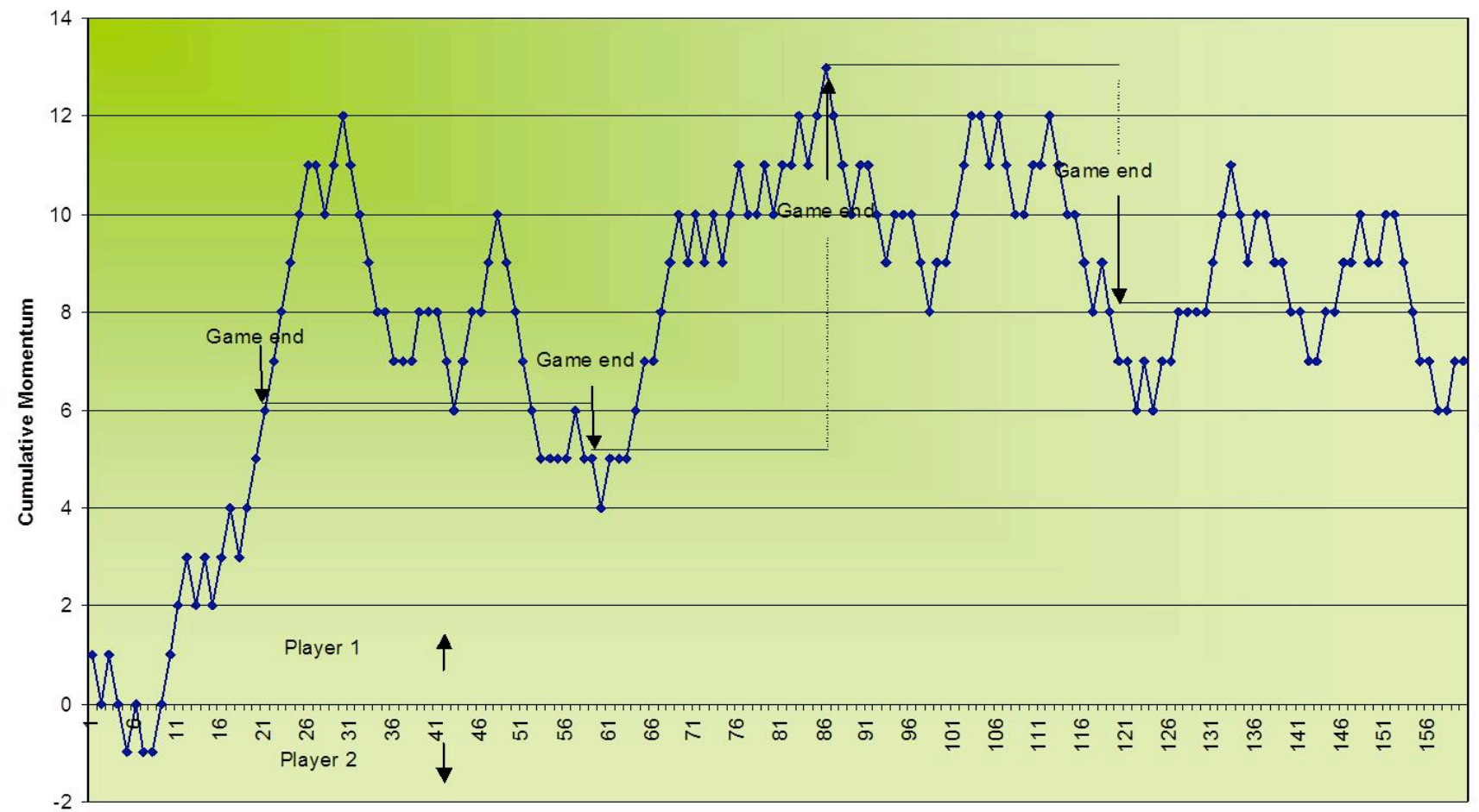

Rallies

Figure 3. Example of a cumulative momentum analysis graph 
Figure 4 is the impressive profile of Player 1, showing a positive increasing curve throughout the match, and a similar profile for Player 2, apart from a big trough at the start of the match. Looking at these 2 profiles, it is difficult to see who won the match and where the turning points occurred. Although Player 2's curve does not reach as high as that of Player 1 at the end, this is not the important factor - it is the slope of the curves that is important, so perhaps we could divine from this that Player 2 won the match. But if we examine the cumulative 'interactive' momentum graph of the two players (Figure 3), the patterns of momentum become much clearer. By marking where the end of each game occurs, it is easy to see that Player 1 won the first game easily, just lost the second, won the third again very easily, but then lost momentum losing the fourth and fifth games. A coach would be very interested in talking about concentration and application at some of these critical points in the match where the momentum shifted so starkly.

In figure 5 the $x$-axis represents the number of rallies in the match. The $y$-axis on the right side represents momentum: $+1,+2$. $-1,-2$, etc... The $y$-axis on the left side represents number of shots in each rally. This corresponds with the dark red vertical bars. So the graph of momentum is now superimposed on to a graphical representation of the respective lengths of the rallies in the match. These data were requested by the physiologist working with the English squash players to see if we could see any fatigue effects - losses of momentum, or other wise, by players after long rallies in the latter stages of a match.

Momentum Analysis - Player 1 v Player 2

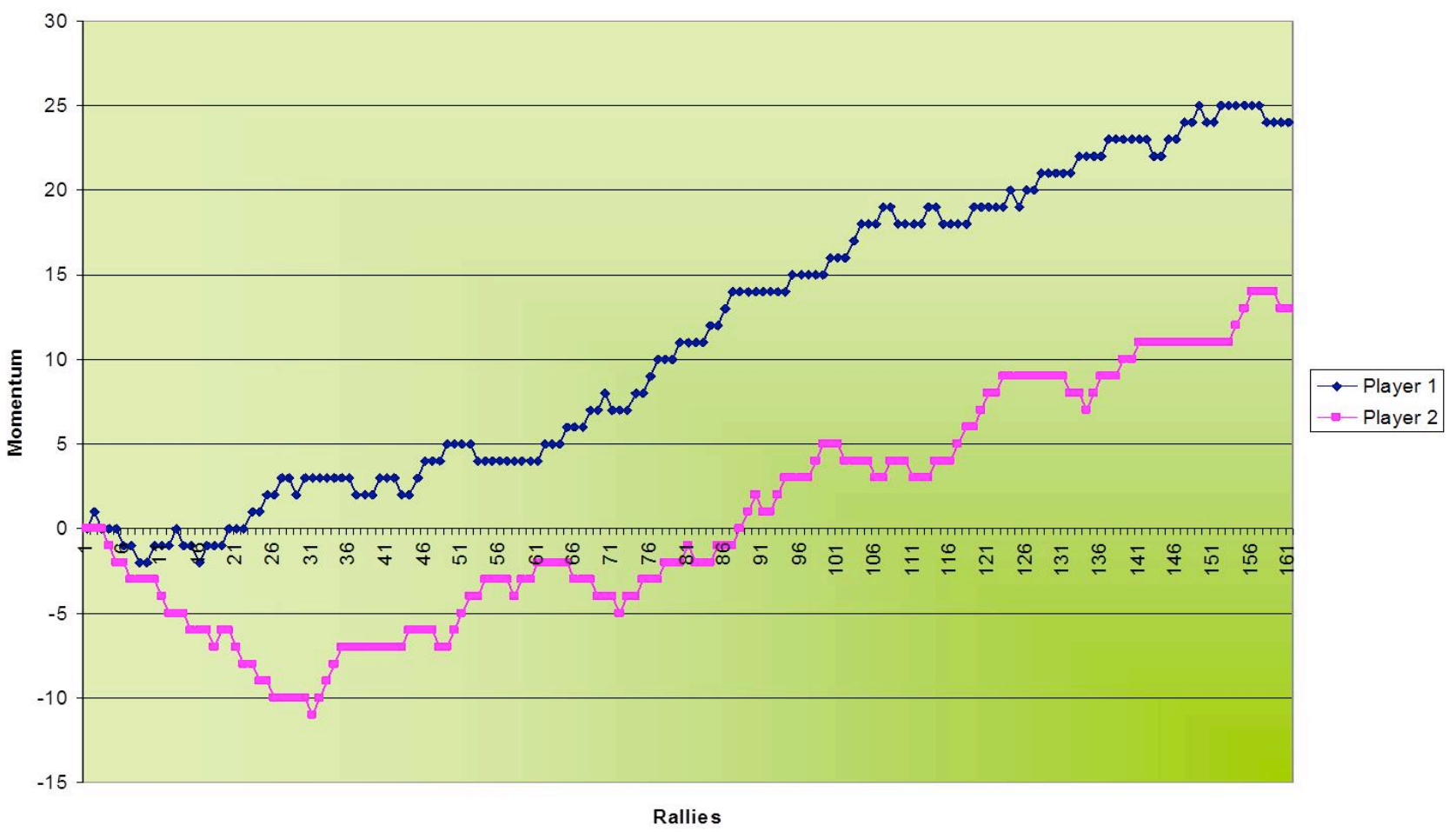

Figure 4. The Momentum graph of 2 players with similar profiles. 


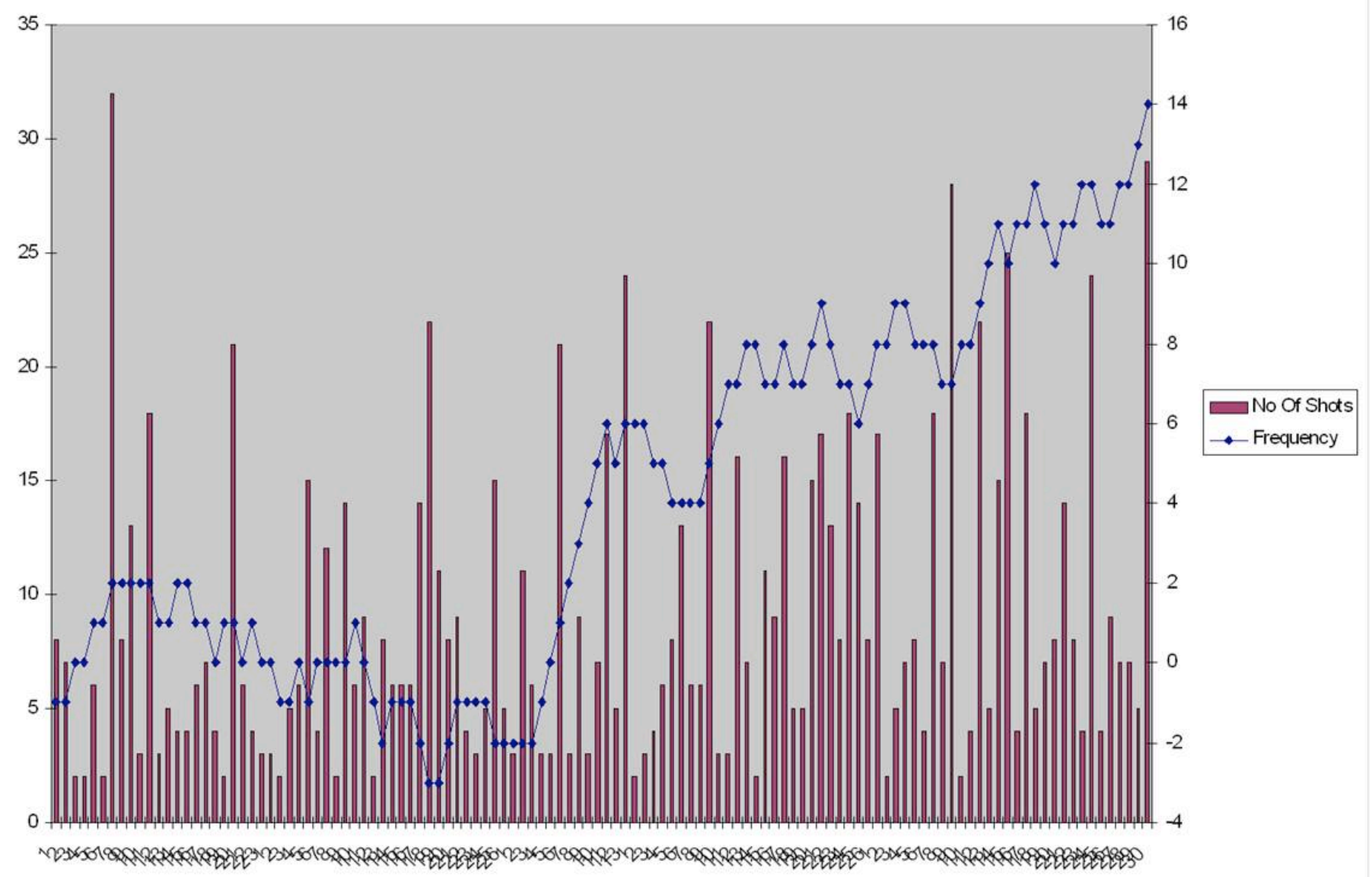

Figure 5. An example of an individual player's momentum with rally length.

\begin{abstract}
Momentum in volleyball
Using post-event analysis Bürger (2009) examined 1066 rallies according to positive, negative or passive momentum. All available sets were either assigned to the successful or the unsuccessful side. The contribution of winners and errors from both sides was almost equal (succ: 534; unsucc: 532). From all tagged games momentum and cumulative frequency graphs (Figure 6.10, Figure 6.11) were compiled. As visual apparentness can not evaluate significant differences between winning and losing performance all data was systematically analysed for successive winners and errors. Positions and lengths of strings of winners and errors were identified using algorithms performed in GNU Octave. Depending on their position these successive winners or errors were assigned to groups forming start $(S)$, middle $(M)$ and end $(E)$ of each set. Therefore the absolute number of points played per set (e.g. 45 points) was divided by three and for example the first 15 points assigned to start the next to middle and the last 15 points to end. This was to detect patterns induced by the different psychological stresses appearing within a set. The shortest streak would be the successive occurrence of two positive or negative actions and the longest one had eight errors in a row. Effectively there were nearly twice as much positive $(21>12)$ and less negative $(75<103)$ strings on the successful teams' side. Differing distributions of streaks no matter if positive or negative could not be found related to the pace of the game. Successful teams' streaks at the beginning (S27), middle (M33) and end (E36) were not significantly different $\left(X^{2}(2)=4.26, p \geq .05\right)$ compared to unsuccessful teams' streaks (S38, M27, E50).
\end{abstract}



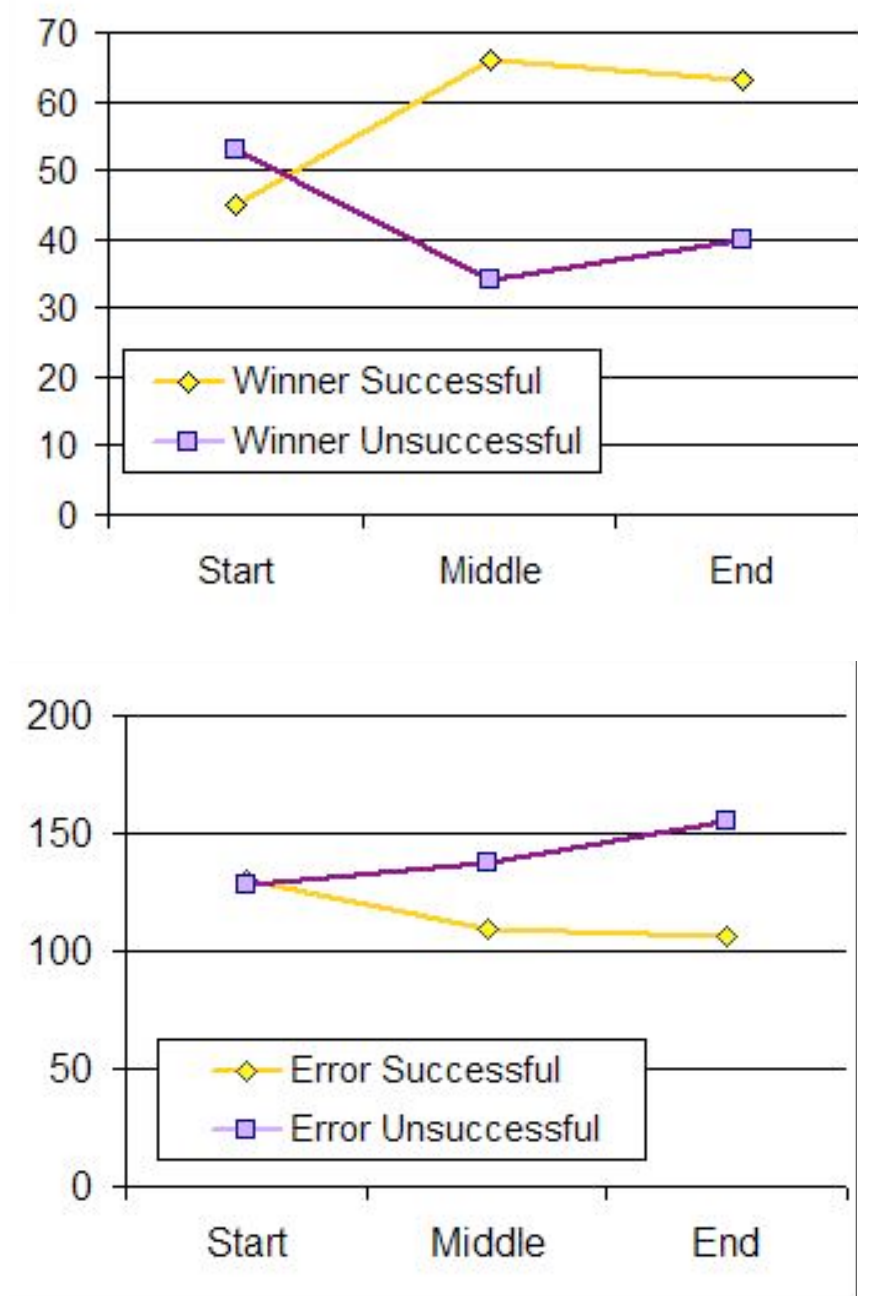

Figures 6 and 7. Development of winners and errors along the game.

It can be stated that there are obvious tendencies towards more and longer strings of positive momentum and shorter and less strings of negative momentum for successful teams at a non-elite level (Bürger, 2009). In relation to the pace of the game winning and losing teams did not show different distributions of streaks no matter if looking at positive or negative streaks. Furthermore using Raab's measure of streakiness (2002) that explore if a winner is more likely to occur after a winner or after an error the rejection of the hot hand hypothesis was the only possible conclusion for the analysed teams within Bürger's research. Still the development and ratios of winners and errors are worth mentioning. Whereas the number of successful teams' winners increased up to a peak in the middle of a set the unsuccessful teams development of winners was contrary (Figure 8). The number of errors made by both sides was evenly high during the first third of a set. However, the direction of development for teams showing an overall bad performance was straight upwards whereas successful teams manage to avoid errors in the pace of a set and to level to the end (Figure 9). Probably the investigation at an elite level of play may give more significantly answers if the maintaining of periods of positive momentum can define a successful team. 


\section{Examining the patterns within the graphs}

Matches of elite squash players ( $N=8$ per player; 6 male and 6 female, all in the top 40 in the world rankings at the time) were analysed to test whether the better players had more positive momentum patterns. This was completed by examining the length of the 'peaks' of momentum in a match, and their corresponding amplitudes, and also comparisons of these 'positive' characteristics to those considered 'negative', that is the 'troughs' of momentum. The peak lengths and heights were measured for each player and averaged; the same process was used for obtaining the averages for each player of their respective trough heights and lengths. Inevitably large variations were found within each player's set of data, but all of these characteristics of the profiles stabilised to within $10 \%$ of their respective means within 6 of the 8 matches, for all the players (see Hughes et al., 2001). A Xi analysis was used for comparison purposes and the patterns showed significant differences between players $(P<0.05)$.

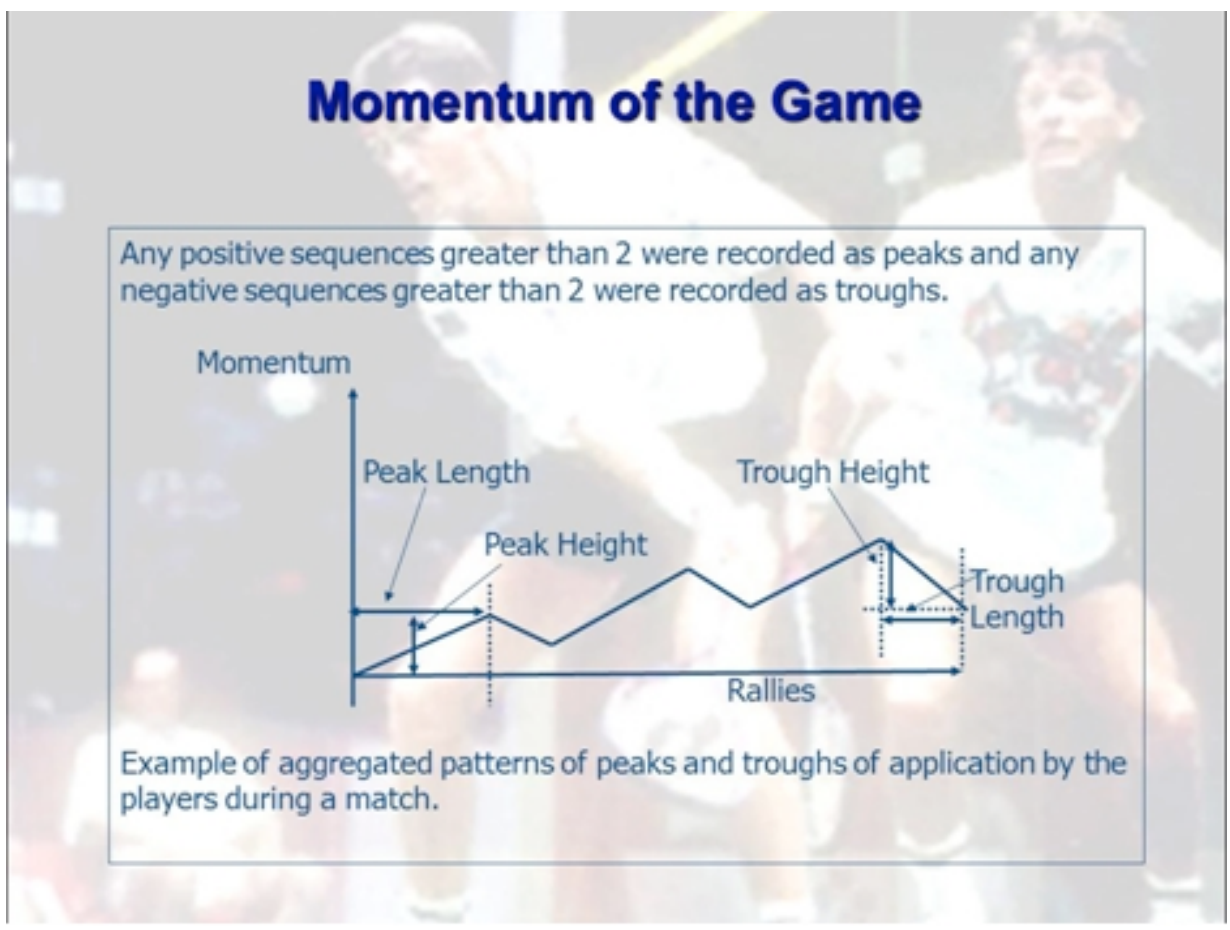

Figures 8. Example of aggregated patterns of peaks and troughs by the players during matches.

The troughs in the data did not show any clear patterns with respect to world ranking, but the lengths of the peaks in momentum showed that, for both men and women, that the world number one's, both men and women, had values well in excess of players below them. These lengths also had an almost perfect correlation $(R=-0.83)$ with the world ranking of the women, this was not so for the men. It was felt that the women's data was more homogeneous than that of the men's, most of the women's matches were against each other, whereas those of the men had a number of the matches against lower ranked players, thus skewing the data for this sort of comparison.

$\mathrm{Xi}^{2}$ analysis was used and showed significant differences between patterns (of peaks, peak lengths, troughs and trough lengths) of the players $(P<0.05)$. Interestingly, the data of the world men's number 2 reflect this particular player's reputation as being brilliant but mercurial, whilst that of the number 1 , clearly demonstrate his application skills, his steadiness, and his desire to dominate his opponents. In the data of some matches of this player, it was observed that he did not make 2 consecutive errors in the whole match. 
Table 1. The lengths and amplitudes of upswings (peaks, of $\geq 2$ ) and downswings (troughs, of $\leq-2$ ) in momentum for 6 elite female and 6 elite male players, averaged over 8 matches for each player.

WOMEN

World

Peaks

Troughs

\begin{tabular}{ccccccccc}
\hline Ranking & Length & SD & Height & SD & Length & SD & Depth & SD \\
$\mathbf{1}$ & 33,4 & 22 & 6,8 & 3,5 & 11,7 & 13,7 & $-4,9$ & 2,5 \\
$\mathbf{2}$ & 30,6 & 16,5 & 8,5 & 4,8 & 14,6 & 9 & $-5,2$ & 1,7 \\
$\mathbf{3}$ & 20,2 & 10,6 & 5,9 & 3,4 & 17,6 & 15 & $-3,8$ & 2,2 \\
$\mathbf{7}$ & 22,4 & 14,8 & 7,1 & 6 & 10,4 & 8,2 & $-2,6$ & 0,6 \\
$\mathbf{8}$ & 14,3 & 12,4 & 4,3 & 2,9 & 11,5 & 9,2 & $-3,2$ & 1,6 \\
$\mathbf{1 2}$ & 15,9 & 10 & 4,9 & 3,4 & 26,2 & 20 & $-3,2$ & 1,8 \\
\hline
\end{tabular}

MEN

World

Peaks

Troughs

\begin{tabular}{ccccccccc} 
Ranking & Length & SD & Height & SD & Length & SD & Depth & SD \\
$\mathbf{1}$ & 45,4 & 28,5 & 9 & 5,7 & 12,9 & 10,3 & $-3,6$ & 0,9 \\
$\mathbf{2}$ & 16,3 & 12,4 & 5,2 & 4,1 & 9,7 & 8,3 & $-3,2$ & 1,4 \\
$\mathbf{7}$ & 20,1 & 20,6 & 5,1 & 5 & 13,5 & 12,8 & $-3,9$ & 2,6 \\
$\mathbf{8}$ & 21,8 & 20,7 & 5 & 4,6 & 14,5 & 10,3 & $-3,8$ & 2 \\
$\mathbf{1 5}$ & 23,4 & 15,8 & 4,4 & 2,8 & 9,9 & 5,7 & -3 & 1 \\
$\mathbf{3 3}$ & 18,9 & 13 & 5,8 & 4 & 15 & 16,4 & $-3,5$ & 2,1 \\
\hline
\end{tabular}

There were many options to manipulate the data, because of the size of the database available. Perhaps data collection limited the findings and accuracy. One suggestion was to collect data from matches where the players were both ranked in the top 20 at the time of the match. However, a player's ranking only tells part of the story. One player may be better than another but has just started playing on tour. Another player might be past his prime and ranked in the top twenty only because he has been playing on tour for such a long time. The male player, ranked 33, had been number 2, is 32 years old and had been injured for some time, and is not playing much now, but when he does compete he is still a very good player. So, retrospectively, he was not a good choice.

This research is a beginning of a new way of extending notational analysis. Studying momentum and linking it to psychological factors could benefit a player's performance. Often players lose concentration or allow themselves to be disturbed by a refereeing decision or the behaviour of their opponent, these will be easily identified by momentum charts, pin-pointing the moment. The players can then discuss the video of these critical incidents with the respective psychologists, hopefully training themselves to better levels of application, like the world number 1's.

This study demonstrates a quantitative, yet qualitative, approach to psychological momentum. Both the world number 1 male and female players have averages of peak length significantly higher than their peers. 
Further research needs to be done to analyse why their peaks are longer and steeper. A questionnaire could be designed to ask players questions about individual matches or overall performance before or after a match. Clips of a previous match can be shown and video and then another questionnaire could be applied.

But in order to provide significant information, these studies need to maintain an interdisciplinary modus operandi. At the current time, few analysts, coaches and athletes are aware of momentum analysis, but this paper attempts to show that momentum can be helpful in performance development. We invite other researchers to explore these applications.

\section{Perturbations in sports match play}

The concept of open (complex) systems is a theory which seeks to explain how regularity emerges from within a system that consists of many degrees of freedom in constant flux. Theoretically dynamic patterns are founded on, and greatly inspired by the pioneering work of Hermann Haken, who introduced the concept of non-equilibrium phase transitions within natural patterns (Haken, 1983). At the heart of this theory is how patterns are formed in complex systems with small changes to the system prompting large (nonlinear) changes in the system. Kelso, Turvey and colleagues have been instrumental in applying these types of theory to the experimental analysis of perception and action (for a review, see Kelso, 1995). In these patterns, contents aren't contained but are revealed by the systems dynamics. Understanding of these theories within brain and behaviour sciences has opened up entirely new research avenues including synergies, cooperation, and control and order parameters.

In the first instance it was recognised that some characteristics of dynamic systems - namely transient periods of instability - were occurring naturally within observed sports performance. McGarry et al., (1995) therefore reasoned, and later confirmed (McGarry et al., 1999) that a disrupting perturbation occurred when the usual stable rhythm of play was disturbed by extreme elements of high or low skill. Using the assertions of Haken (1987) it became clear to those researchers following McGarry, that the analysis of perturbations in sport offered a more critical and dynamic method of investigation and therefore a significant step towards effective support to coaches and performance.

During the last decade, studies using these and related theories have attempted to provide a theoretical basis to sports - and most relevantly, performance analysis - research. Understanding has been sought in terms of feature identification (Ferreira et al., 2003; Palut \& Zanone, 2003) and essential variables which characterise pattern formation (Hughes \& Reed, 2005; Palut \& Zanone, 2005). However our understanding of these and other critical behaviours of sports systems remains in its infancy.

Match play sports exhibit rhythms when competitors perform at equal levels. A perturbation exists where the usual stable rhythm of play is disturbed by extreme elements of high or low skill. In soccer, should the resulting instabilities in playing patterns lead to a shot on goal, then the outcome is termed a critical incident.

"If we study a system only in the linear range of its operation where change is smooth, it's difficult if not impossible to determine which variables are essential and which are not".

"Most scientists know about nonlinearity and usually try to avoid it". 
"Here we exploit qualitative change, a nonlinear instability, to identify collective variables, the implication being that because these variables change abruptly, it is likely that they are also the key variables when the system operates in the linear range".

(Kelso, 1999)

Research confirming the existence of perturbations by McGarry \& Franks (1995) in squash identified particularly weak or strong shots that place one player at a recognised disadvantage to another.

Squash lends itself strongly to the dynamical system theory as the game is always played between two players; therefore the behaviour of one directly affects the behaviour of the other (Hughes et al., 2007). McGarry et al. (1999) began to analyse squash as a dynamical system, first by investigating whether the system can be detected as switching between periods of stability and instability from visual inspections. Independent observers were able to identify those behavioural transitions, or perturbations, that were held as switching the system from and to regions of stability and instability. The identification of perturbations from visual inspections of soccer behaviour was likewise reported by Hughes et al. (1997). These ideas are explored in detail by Murray et al. (2008).

Many sports can therefore be seen as an open system that exists in a dynamic equilibrium or stable state (McGarry \& Franks, 1995). An open system displays rhythmical patterns and often displays invariant behavioural. In order to control the rhythm of the game, it is essential to attempt to keep your opponent under pressure and not in control of the centre of the court, called the ' $T$ '. When a disturbance in a dynamical system occurs, it is defined as a perturbation - in squash this is a very good shot that therefore upsets the rhythm of the rally and puts physical pressure on the opposing player. Hence perturbations exist in an open system where the normal constant rhythm of play is disturbed by excessive elements of high, or low, skills. This consequently results in a particular outcome (Hughes \& Reed, 2005), which places one of the players, or a team, at a disadvantage, for example their displacement on court.

Match play in squash alternates intermittently between stable and unstable behaviour, it is at the boundaries or transition points of these behaviour states that 'critical incidents' are most likely to be detected (McGarry et al., 2002). When perturbations arise they do not all lead to critical incidents, which places a player under severe pressure, though, these critical incidents can be overcome by a defensive shot or sharp movement that will re-establish the rally's stability.

Hughes et al. (2000a) had suggested that concentrating upon the critical aspects of soccer play "could make the ensuing analyses not only easier but more relevant". A perturbation in soccer is hence defined as an incident that changes the rhythmic flow of attacking and defending; for instance a penetrating pass, change of pace or any moment of skill that creates a disruption in the defence.

Hughes et al. (2000) attempted to confirm and define the existence of perturbations in association football; twelve common attacking and defending variable were identified from English football that led to scoring opportunities. These twelve causes were shown to occur consistently, covering all possible eventualities and had high reliability. After further analyses of the 1996 European Championship matches $(\mathrm{N}=31)$, Hughes et al. (2000b) attempted to create a profile for nations that had played more than five matches. Although the work supported English League traits for successful and unsuccessful teams, there was insufficient data for the development of a comprehensive normative profile. Consequently, although failing 
to accurately predict future performance, Hughes et al. (2000b) had introduced the method of using perturbations to construct a prediction model, and had identified 6 attacking skills and 6 defensive skills that contributed most to the creation of perturbations.

In match play, teams may alter tactics and style according to the game state/nature; for instance a team falling behind may revert to a certain style of play to create more goal-scoring chances and therefore skew any data away from an overall profile. Consequently, although Hughes et al. (2000) had classified perturbations, the method had failed to distinguish between the game states, which prevented the generation of stable and accurate performance profiles.

In some instances, a disruption in the rhythm of play may not result in a shot, owing to high defensive or a lack of attacking skill. Developing earlier work on British league football, Hughes et al. (2001b) analysed how international teams stabilised or 'smoothed out' the perturbation. Hughes et al. (2001b) identified 3 categories of perturbation that did not lead to shots on goal; actions by the player in possession, actions by the receiver and interceptions. Although Hughes et al. (2001b, p.32) highlighted the requirement for improved technical skill, by combining the data of many teams the conclusions provided little benefit for coaches of specific teams and highlighted the need for analysis of individual "team 'signatures' ".

Rugby Union, whilst posing many of the same structural characteristics as football, is arguably a more complex sport. This complexity of performance often leads to a huge amount of data being collected from a single performance, making its analysis and relevance often extremely confusing to both coach and performer. And with the advent of systems such as ProZone, we continue to expand the depth and range of data available to the professional coach; therefore relying upon largely subjective decisions to ration this information.

The work of Locke (2005) to successfully identify perturbations within Rugby Union has begun this process of data 'concentration'. However these methods require further development and application; Rugby, as a result of one its most fundamental rules (the backward pass) produces a significant number of lateral phases and patterns of play, which may serve to disperse or hide skill perturbations. Such is the nature of the game that the perturbation instance itself may be caused by an earlier disruption within a separate phase of play; for instance backs committing to a breakdown leaving forwards exposed on the outside. It is therefore suggested that dynamical methods within team sports are more relevant when examining the phase of play rather than instant of occurrence - of which Locke (2005) identified 18.

Within Rugby Union a player can only pass the ball backwards or laterally (i.e. not forward) to another player, or kick it. This means that the majority of progress made by an attacking team occurs through repetitive cycles of passing the ball, running to make ground and being tackled. Each of these cycles (greatly simplified) is called a phase of play. Teams within top-flight Rugby Union possess very well organised defences, which tend to produce stable phases of play which can continue for significant periods of time. Although, as Locke (2005) suggests, it can appear that neither team appears to be in definite control of a particular phase, the outcome of that phase is - more often that not - a positive or negative for the team in possession.

Supported by basic coaching knowledge, it is proposed that phases of play can be categorised into positive, negative or 'neutral' moments. It is a simple step to suggest therefore that teams who maintain consecutive, positive 'phases of play' (what will later be termed momentum), will be more likely to create 
significant disruptions in the defence (perturbations) which in turn lead to scoring opportunities. Reed and Hughes (2005) suggested the following arguments against skill/instant perturbations:

- Teams within top-flight sport possess very well organised defences, which tend to produce stable phases of play which can continue for significant periods of time... contests exhibiting a general tendency to stability.

- Such is the nature of many sports, that the perturbation instance itself may be caused by an earlier disruption within a separate phase of play. For instance:

- Rugby Union backs committing to a breakdown leaving forwards exposed on the outside.

- Soccer centre back losing his positioning which stretches the defence elsewhere.

- Perturbations become 'hidden' or confused by a multitude of connecting variables and factors.

Figures 9 and 10 show the analyses of 2 under- 16 International Rugby Union matches (producing $~ 360$ bits of data) notated using SportsCode Elite, post-event, and using this 'momentum of positive/negative phases of play' approach. Essentially phases of play were classified into 3 categories (positive, negative and neutral), but with subcategories to provide coaching feedback. Subsequent data analysis used basic algorithms to convert SportsCode edit list data into numerical vales for presentation and analysis purposes. In this instance a positive phase equated to +1 , negative, -1 and neutral 0 . Perturbations and scores (for and against) were notated, with corresponding times.

Figure 9 shows the interaction between the 2 teams, with positive momentum for Wales (negative for England) being indicated as the curve moves above the abscissa, positive momentum for England (negative for Wales) is shown as the curve plunges downwards - so it is the slope of the curve that shows the current state of momentum. In this example Wales started well (10 mins), had a fairly level patch for about 20 mins and then finished the half very strongly. The second half was not so good - in fact the metaphor 'all down hill' fits exactly.

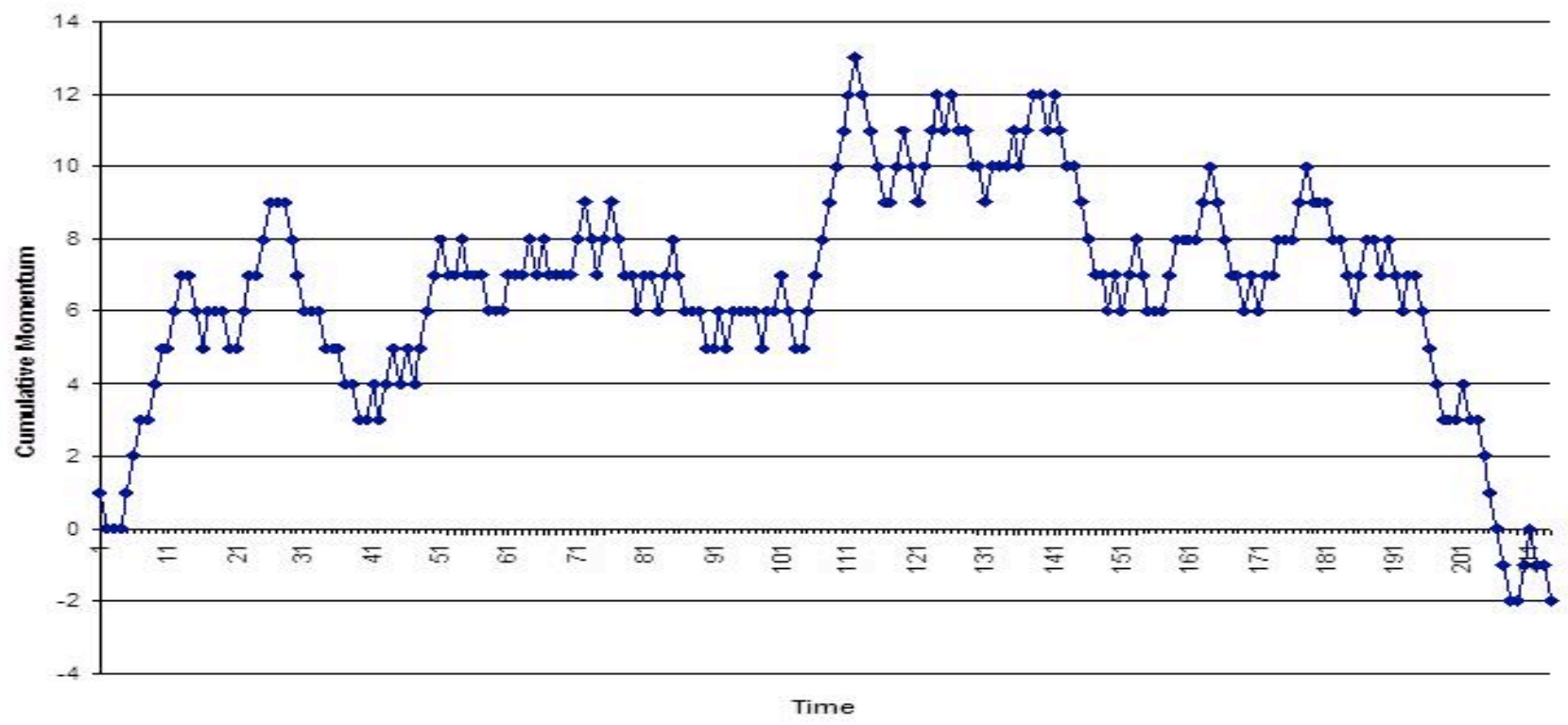

Figures 9. Cumulative momentum plot for match 1 


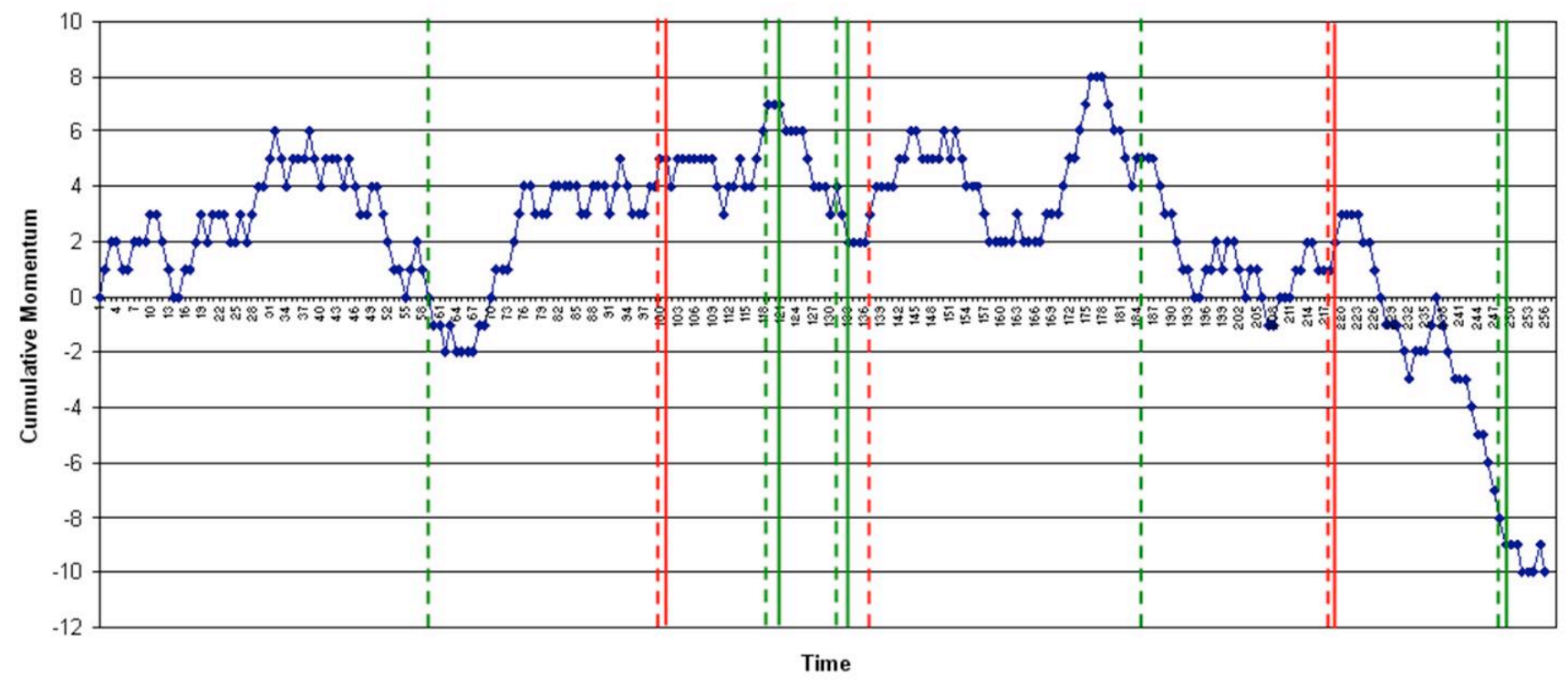

Figures 10. Match 2 -Cumulative total phase scores with perturbation indicators

In the figure 10 the scores have also been indicated. This match shows a similar falling away of momentum by the Welsh team. But if the team analyst had an in-event system of this nature, it would be clear that changes needed to be made, in spite of the Welsh try 10 mins from the end. The subsequent substitutions might have prevented the final match winning try by the English team 2 mins from the end. Any coaches in any team match play sports can probably translate these examples to their own sports.

- Reed \& Hughes (2006) have considered momentum to be the cumulative effect of phases of play; simply, each phase contributes towards a team's momentum.

- Whilst some evidence has been presented to substantiate these claims, these data serve only to introduce the subject, in a crude and simplistic fashion.

- However the ability of these methods to portray a great-deal of information simply and effectively, whilst retaining fundamental performance and dynamic information provide significant advantages over traditional notation methods.

- As we have seen, these data can act as a powerful first point of reference - and with further development may enable:

- Immediate interventions based on momentum profile.

- Profiling.

- Although the published evidence in favour of momentum (psychological or otherwise) is ambiguous at best, coaching resources continue to refer to the subject extensively (Higham, 2000) thus suggesting that many of our existing notation methods based around scoring and performance indicators are inherently flawed.

- For example momentum can be gained (and lost) without points or goals being scored, as demonstrated within this paper. This is precisely the reason that the score does not always reflect the state of play, and why the better or stronger team or player does not always win.

- The data presented within this paper serve to introduce the concept and illustrate the potential of dynamic momentum analyses. It is hoped therefore, that future research develops the points raised within this study, enhancing our understanding and procedures of analysis. 
So far research on momentum has been mostly limited to individual performance either in basketball, baseball, billiard, squash, tennis and so forth. The question arises whether momentum is transferable to team games like volleyball or not. Moreover are sequences of winners and errors, represented as momentum graphs, able to detect differences within the pace of the game?

An outstanding performance from one player like an ace or a point scoring attack may transfer positive momentum to the rest of the team. Herbert (1991) stated that the seconds before the serve in volleyball are a very critical moment. Uncertainty of one player can develop to a full blown anxiety attack influencing the whole team. He describes this phenomenon from his own point of view as a women's coach as followed:

The dead-ball period leading up to the whistle that initiates the serve produces, in my opinion, more anxiety than any other time during competition. In all other phases of the game, players are moving, reading, and reacting to the multiple, changing cues that characterize the rapid flow of play during rallies. Their minds are occupied by with the second-by-second demands of keeping up with the pace of the game. But in this one instance before play begins, with players standing in place, waiting for the triggering effect of the serve, negative and unproductive thoughts can invade the receiving team's side of the net $[\ldots]$ (Herbert, 1991).

This shows that negative momentum is even more threatening and therefore need to be avoided in individual sports and even more in team games. Bürger (2009) explored the ides of momentum in volleyball.

Momentum and perturbations in other team sports

Performance profiles are suggested to be a description of a pattern of performance from an analysed team or individual (Hughes et al., 2001). The early work of Sanderson and Way (1977) and Hughes (1986) highlighted that the formation of a database of matches that provide information regarding patterns of play that could be considered representative of the subject used to form the database. Through these databases, players and coaches may use the findings to prepare and analyse individual's performance before playing the match. This is now a formidable tool in a player's or team's preparation and performances (Murray \& Hughes, 2001).

Momentum is a physical term that refers to the quantity of motion that an object has and can be defined as 'mass in motion'. Murray \& Hughes (2001) were the first to introduce the concept of momentum analysis in squash, and from this analysis, players were able to see their momentum through the match - these data curves were calculated using the rally's ending shot, positive changes for winners, negative changes for errors.

Hughes et al.'s (2006b) study of momentum in elite players shows there is conclusive evidence that management of this momentum by elite squash players correlated strongly with the world rankings of the players. Hughes et al. (2006b) examined the winners and errors of a number of players' matches from which they calculated each player's momentum profile. It is recognised by most coaches in squash, that it is not the last shot in the rally that is the most important, hence the complex rally analyses of Sanderson \& Way (1977) and most subsequent researchers (Hughes, 1986; Hughes \& Robertson, 1998; Hughes et al., 2000). By recording the perturbations in rallies, the 'pressure shots' are being entered into the database, it seems logical then that profiles drawn up on these shot data will present more informed ways in which a 
player puts his or her opponents under pressure, and conversely, how they themselves react to these types of pressure situations.

The function of the investigation by Murray et al. (2008) was to assemble momentum profiles of elite male and female squash players by using perturbations in performance, and compare these with momentum profiles calculated from the same matches using winners and errors. Information from these profiles will then be compared to those profiles gathered by more traditional means (Murray \& Hughes, 2001).

The way in which the players use perturbations within their match play can be identified from the momentum graphs. The more defensive usage of Ricketts and Lincou can be distinguished from their similar graph patterns. Whereas the more attacking style of Palmer detects a different pattern that emerges within the momentum graphs. From the case studies, it assumed that the momentum graphs using perturbations do not illustrate any representation to whether a player won the match as identified from the winner error momentum graphs produced from this study and Hughes et al's (2006b) work on momentum. The case study approach identified that the use of perturbations in calculating momentum, in conjunction with momentum graphs from winners and errors, produce another form of representation of how players perform. It is felt however, that the sequential nature of the data and the interaction of the players, inhibit the normalisation of these data - in so doing the unique 'signature' of each performance would be lost. But, from the perturbation momentum graphs, there is clear evidence that these patterns do complement the traditional methods of creating profiles which should enable coaches to analyse in more depth the performance of players, and their opponents, and to forge future game plans.

\section{Momentum plot (W and $E$ ) for TL against DP}

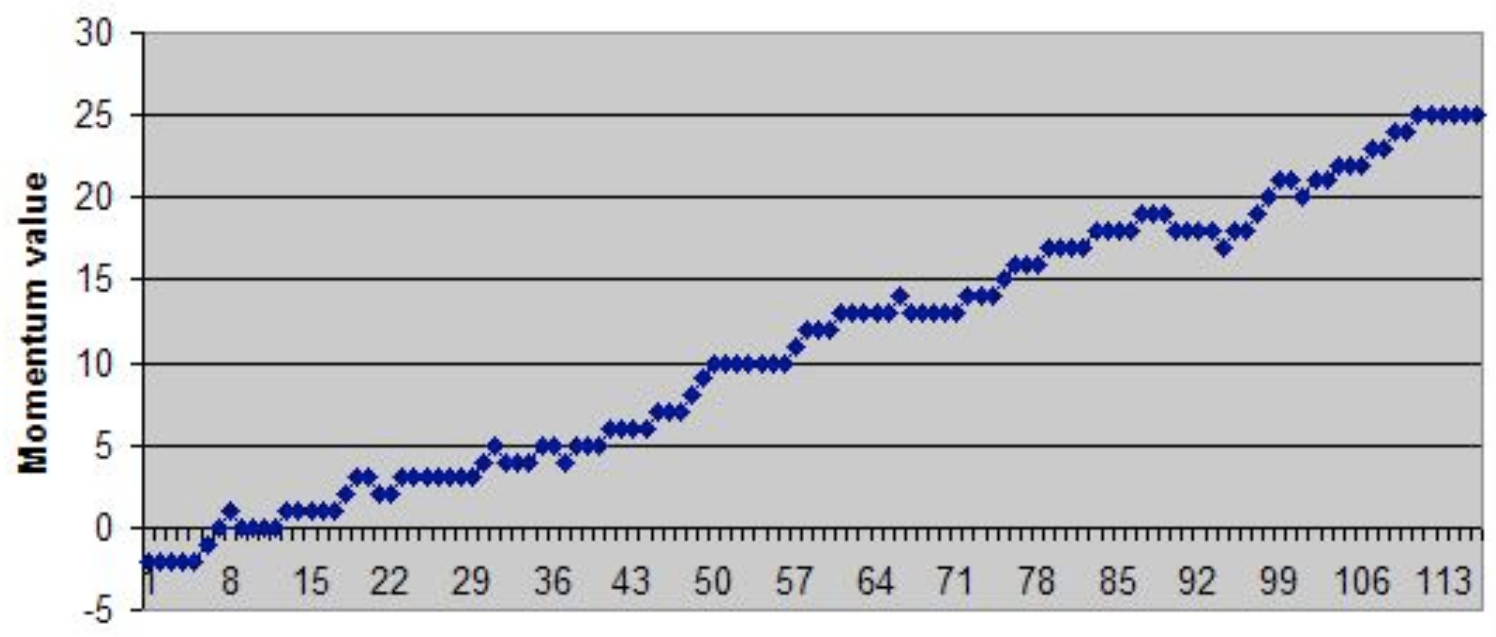

Rally number

Figures 11. The winner and error Momentum graph of TL in his match with DP 


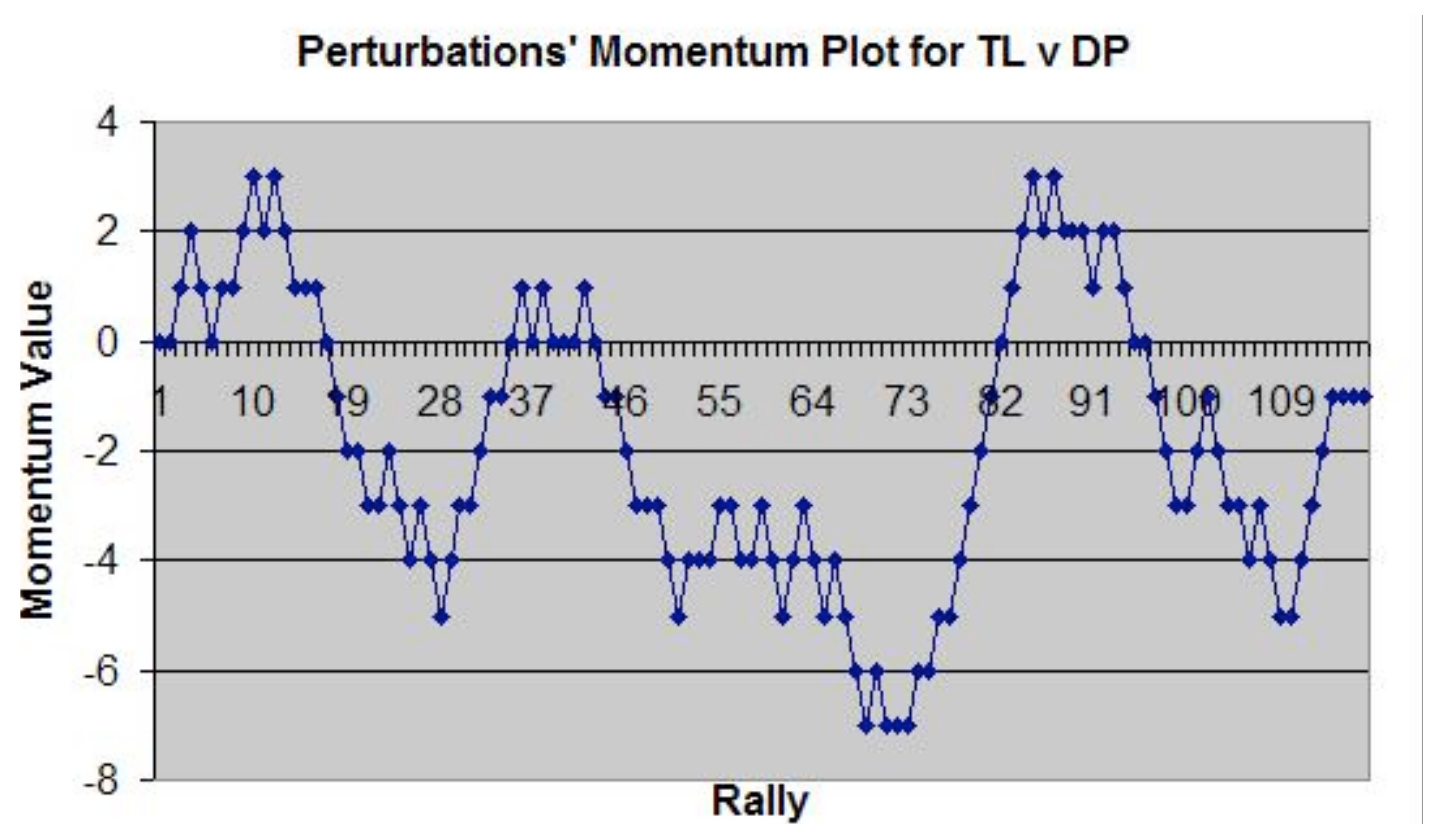

Figures 12. The perturbations' Momentum graph of $T L$ in his match with DP

It was concluded that there was shown to be no comparison between the momentum using winner and errors, to the momentum using perturbations. Nevertheless, after conducting a case study on some of the subjects, indications of usage patterns of perturbations within matches started to emerge form the momentum graphs, which identified specific styles of play. Adding the data from different matches was deemed to be counter-productive to the true value of using these graphs and also the sequential nature of the data involved. There is evidence to indicate that momentum using perturbations can further the analysis in squash. This study also concluded that the physiological characteristics of physical conditioning has an effect upon the amount of perturbations caused. This could be attributed to a player becoming more urgent towards the end of a match and performing more high risk shots, as evident through rally lengths imposed with the momentum graphs using perturbations.

Further research into the points of critical momentum shift could be investigated to whether situations of the match, for example refereeing decisions, caused the some critical swings in momentum within matches. This could be analysed qualitatively by interviewing the players and coaches after the match to see whether there where areas of the match where they thought that the momentum shifted and for what reasons. From the results the interviews could be analysed correspondently with the momentum graphs to identify and confirm the critical incidents within the match and what created momentum instabilities. An extension of this research could also involve an alternative method for identifying perturbations. Rather than a trained analyst recognising them by eye it could be possible to use tracking hardware or software to highlight them automatically using kinematics variables.

\section{CONCLUSIONS}

Momentum analysis, through notational analysis techniques, has been enthusiastically received by coaches in a number of sports, both individual and team. It enables a clear indication of the turning points in the match, and is particularly interesting when used with qualitative factors, such as positive phases 
(rugby union or basketball) or perturbations (squash, soccer). These researches have been developed with the primary aim of expanding the profiling repertoire of performance analysts in their quest of supporting the coaches and their athletes.

It should be emphasised that the aims of the researchers in this area are very different to the aims of the researchers in 'hot hands' or 'streakiness' analysis, although involved in very similar concepts and consequently their analytical procedures and statistical processes are therefore different.

\section{REFERENCES}

1. BÜRGER P. Perturbations and momentum analysis of volleyball. MSc Dissertation. UWIC. 2009.

2. FERREIRA PF, VOLOSSOVITCH A, GONÇALVES I. Methodological and Dynamic Perspective to Determine Critical Moments on Sport Game. International Journal of Computer Science in Sport. 2003; 2(2): 119-122.

3. HAKEN H. Synergetics, an introduction: Non-equilibrium phase transitions and self-organisation in physics, chemistry and biology. Berlin: Springer, 1983.

4. HEBERT M. Insights and Strategies for Winning Volleyball. Campaign, III.: Human Kinetics, 1991.

5. HUGHES M. A comparison of the patterns of play of squash. In ID Brown, R Goldsmith, $K$ Coombes \& MA Sinclair (Eds.), International Ergonomics. London: Taylor \& Francis. 1985:139-141.

6. HUGHES M. A review of patterns of play in squash. In Sports Science (edited by J Watkins, T Reilly \& L Burwitz). London: E\&FN Spon. 1986: 363-368.

7. HUGHES M, REED D. Creating a performance profile using perturbations in soccer. In: Proceedings of 4th International Scientific Conference on Kinesiology, edited by D. Milanovic \& F. Prot. Zagreb: University of Zagreb, Croatia, September. 2005: 34-53.

8. HUGHES M, ROBERTSON C. Using computerised notational analysis to create a template for elite squash and its subsequent use in designing hand notation systems for player development. In A. Lees, I Maynard, M Hughes \& T Reilly (eds) Science and Racket Sports II. London: E. and F.N. Spon. 1998: 227-234.

9. HUGHES M, DAWKINS N, DAVID R. Perturbation effect in soccer. Notational Analysis of Sport III, Cardiff: CPA, UWIC. 2000a: 1-14.

10. HUGHES M, DAWKINS N, LANGRIDGE C. Perturbations not leading to shots in soccer. In M Hughes (ed.) Notational Analysis of Sport III, Cardiff: CPA, UWIC. 2000b: 108-116.

11. HUGHES M, EVANS S, WELLS J. Establishing normative profiles in performance analysis. EIJPAS International Journal of Performance Analysis Sport. 2001; 1:4-27.

12. HUGHES M, FENWICK B, MURRAY S. Expanding normative profiles of elite squash players using momentum of winners and errors. EIJPAS International Journal of Performance Analysis Sport. 2006; 6(1): 145-154.

13. HUGHES M, HUGHES MT, BEHAN H. The evolution of computerised notational analysis through the example of racket sports. International Journal of Sports Science and Engineering. 2007; 1: 328

14. KELSO JAS. Dynamic patterns: The self-organisation of brain and behaviour. Cambridge, MA: MIT Press, 1995.

15. LOCKE D. Perturbations in team sports: How rugby union fits a dynamical systems model. Unpublished MSc dissertation. Wales: UWIC, 2005.

16. MCGARRY T, FRANKS IM. Modelling competitive squash performance from qualitative analysis. Human Performance. 1995; 8(2): 113-129. 
17. MCGARRY T, FRANKS IM. In search of invariant athletic behaviour in competitive sport systems: An example from championship squash match-play. J Sports Sci. 1996; 14(5): 445-456.

18. MCGARRY T, ANDERSON DI, WALLACE, SA, HUGHES M, FRANKS IM. Sport competition as a dynamical self-organizing system. J Sports Sci. 2002; 20(10): 771-781.

19. MCGARRY T, KHAN MA, FRANKS IM. On the presence and absence of behavioural traits in sport: An example from championship squash match-play. J Sports Sci. 1999; 17(4): 297-311.

20. MURRAY S, HUGHES M. Tactical performance profiling in elite level senior squash. In (eds. M. Hughes and I.M. Franks) pass.com, Cardiff: CPA, UWIC. 2001.185-194.

21. MURRAY S, HOWELLS M, HURST L., HUGHES MT, HUGHES MD, JAMES N. Using perturbations in elite men's squash to generate performance profiles. In A. Hoekelmann \& M. Brummond (eds.) Performance Analysis of Sport VIII, Magdeburg: School of Sport, Otto von Guericke Universitat. 2008: 98-115.

22. PALUT Y, ZANONE PG. Tennis investigation as a nonlinear complex system. International Journal of Computer Science in Sport. 2003; 2(2): 132-134.

23. PALUT Y, ZANONE PG. A dynamical analysis of tennis: Concepts and data. J Sports Sci. 2005; 23(10): 1021-1032.

24. REED D, HUGHES M. An exploration of team sport as a dynamical system. In Dancs H, Hughes M \& O'Donoghue P (eds) Notational Analysis of Sport - VII, Cardiff: UWIC, 2006: 63-72.

25. SANDERSON FH, WAY KIM. The development of objective methods of game analysis in squash rackets. Br J Sports Med. 1979; 11(4): 188. 